\title{
Antibiotic usage in Intensive Care Units of Tabriz Imam Reza Hospital, 2011
}

\author{
${ }^{1}$ Sepehr Taghizadeh, ${ }^{1}$ Mehdi Haghdoost, \\ ${ }^{2}$ Omid Mashrabi and ${ }^{3}$ Zahra Zeynalikhasraghi \\ ${ }^{1}$ Infectious Diseases and Tropical Medicine Research Center, \\ Faculty Medicine, Tabriz University of Medical Sciences, Tabriz, Iran \\ ${ }^{2}$ Resident of Internal Medicine, Department of Internal Medicine, \\ Faculty of Medicine, Tabriz University of Medical Sciences, Tabriz, Iran \\ ${ }^{3}$ Department of General Physician, Faculty of Medicine, Tabriz University of Medical Sciences, Tabriz, Iran
}

Received 2013-11-05, Revised 2013-10-21; Accepted 2013-11-22

\begin{abstract}
Drug therapy is one of the most important factors in providing community health. In a proper selection of an antibiotic for curing an infection, the kind of infective agent and its sensitivity to the utilized drugs must be regarded. The patients whom are admitted in ICUs are 5 to 7 folds more vulnerable to infection. The studies has been done in past decades have shown various amounts of inappropriate antibiotic use. The files of 234 patients whom were admitted during 6 months in ICUs (Surgery, Neurosurgery, Neurology and Pulmonary diseases) of Imam Reza Hospital of Tabriz County studied for manner of antibiotic therapy, antibiotic changes during therapy, number of prescribed antibiotics $64 \%$ of patients were male and $36 \%$ were female. Average age of patients was $43.9 \pm 23.9$ years. The most admission of patients was in Surgery ICU (69.2\%). The most prescribed antibiotic was Cephalosporin family and ceftriaxone was the most utilized antibiotic. In $59.7 \%$ of patients, the antibiotics were prescribed empirically and in 35\% of cases antibiotics changed during treatment. $28.1 \%$ of cases treated with monotherapy while others treated with combination therapy. In $61 \%$ of cases, the prescribed antibiotics were inappropriate with final diagnosis of patients. The majority of antibiotics were prescribed according to physician's clinical experiment and combination therapy is used much more than monotherapy.
\end{abstract}

Keywords: Antibiotic, ICU, Cephalosporins

\section{INTRODUCTION}

Medical therapy is one of the routines in treatment of the patients so the logical administration of drugs is one of the most important contributing factors providing the health of the communinty. Antibiotics are of most important drug agents with great role in health providing, if not administrated logically beside its useful effects, these agents can harm the health of the communities and humans with their side effects (Diaz-Martin et al., 2012).
In the proper choice of an antimicrobial agent in the treatment of certain infections, the nature of the infective agent and the drugs used, the sensitivity of the infective agent should be considered. In addition, host factors such as age, location of infection, a history of previous sensitivity to antimicrobial compounds, genetic or metabolic abnormalities, pregnancy and liver and kidney function are important. Patients admitted to the Intensive Care Unit (ICU) suffers from serious hospital infections, 5 to 7 times more than patients admitted to other (Leibovici et al., 1998).

Corresponding Author: Sepehr Taghizadeh, Infectious Diseases and Tropical Medicine Research Center, Faculty Medicine, Tabriz University of Medical Sciences, Tabriz, Iran 
Most of human infections, in the normal health situation can be treated with an antimicrobial agent but due to better antimicrobial coverage of combination therapy, some physicians prefer the combination therapy with 2 or 3 antibiotics but it should be considered that inappropriate use of such therapies can result in adverse effects (Leibovici et al., 1998).

Infections, the most common problem in ICU patients is associated with high mortality, morbidity and costs. So one of the most widely consumed drugs in the ICU are antibiotics (Vincent et al., 2009). Studies show that over $70 \%$ of ICU patients receive antibiotics. Despite the important role of antibiotics in treating the infections, they are involved in the antibiotic resistances due to empirical usage of antibiotics in mentioned hospital wards (Kolle, 2001; Krivoy et al., 2002; Roder et al., 1993; Rice, 2003).

The studies that have been conducted over the past decades had proven varying degrees of incorrect use of antibiotics (Roder et al., 1993). Increasing emergence of antibiotic resistance and threats the recovery of patients while increasing the overall cost of health care costs (Rello, 2007).

Because of the importance and sensitivity of antibiotic therapy in the ICU, this applied study is conducted in the to the general surgery, neurosurgery, brain surgery and Pulmonary ICU wards of Imam Reza (as) Hospital, Tabriz University of Medical Sciences in the first six months of 2011 the aim of this study was to achieve awareness of antibiotic therapy to create a more constructive interaction between different medical groups in the realization of the optimal use of antibiotics.

\section{MATERIALS AND METHODS}

In a cross sectional descriptive applied study in the department of infectious diseases in Tabriz university of Medical Sciences on ICU patients of Imam Reza hospital, the antibiotic therapy methods was evaluated.

A number of 260 files of patients admitted to general surgery, neurosurgery, brain surgery and Pulmonary ICU wards of Imam Reza (as) Hospital were randomly selected. The antibiotic therapy beginning(clinical findings, clinical findings beside microbiologic findings), the changes of antibiotic therapy and its effect on therapeutic process, the antimicrobial combination, duration of antibiotic therapy, the appropriateness of antibiotics and the final diagnosis of patients with underlying diseases were studied. Because of some defects in patient's files, 234 files were finally studied. In this study, no additional intervention was performed on patients, as well as all information, the name and address of the patient is not mentioned anywhere.

\subsection{Analysis of Results}

Data obtained from the records were analyzed using statistical methods (percentage and mean \pm SD) and 18 SPSS statistical software.

\section{RESULTS}

Amongst 234 patients, $64 \%$ were male and $36 \%$ were female, the mean age of male patients was $42.04 \pm 24.32$ years and the mean age of females was $39 \pm 23.28$ years $(\mathrm{p}=0.353)$ (Fig. 1).

About 165 patients was admitted to Surgery ICU, 30 patients $(12.8 \%)$ in Neurosurgery ICU, 21(9\%) in ICU and $18(7.7 \%)$ in Neurology ICU. With considering the portion of antibiotics used in empirical method (49\%) to the percent of the overall administrated antibiotics $(82 \%)$ the percent of empirical administration was $59.7 \%$.

In just $39 \%$ (57 out of 192), the antibiotic administration was appropriate with final diagnosis and in $61 \%$ of cases the antibiotics was administrated inappropriately.

The percentage of antibiotic applies and the method of its beginning is shown on Table 2 .

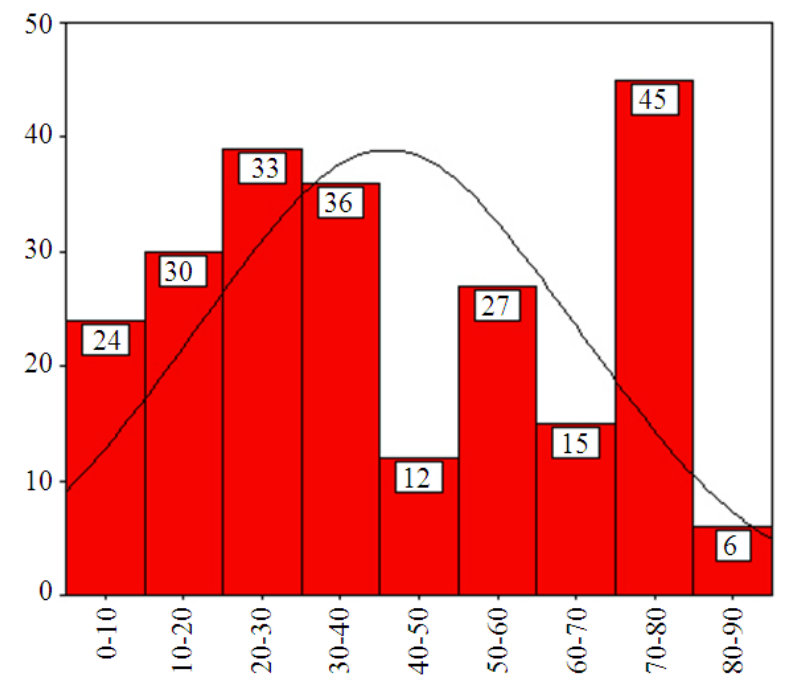

Fig. 1. Age distribution of patients 


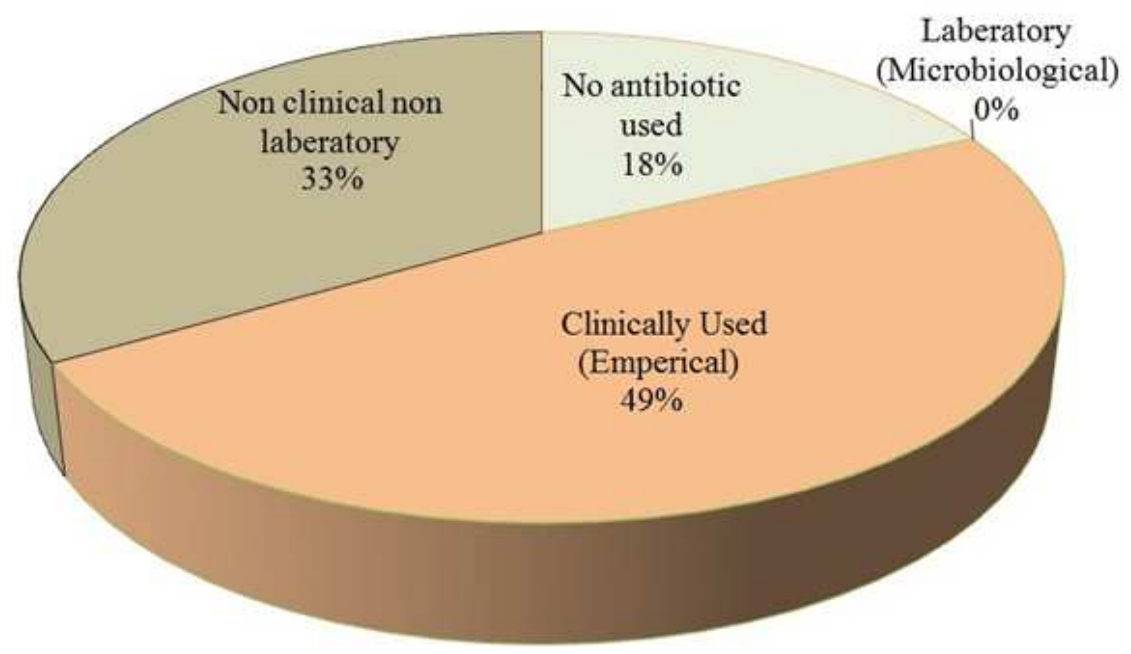

Fig. 2. The frequency of antibiotic administration and how it began

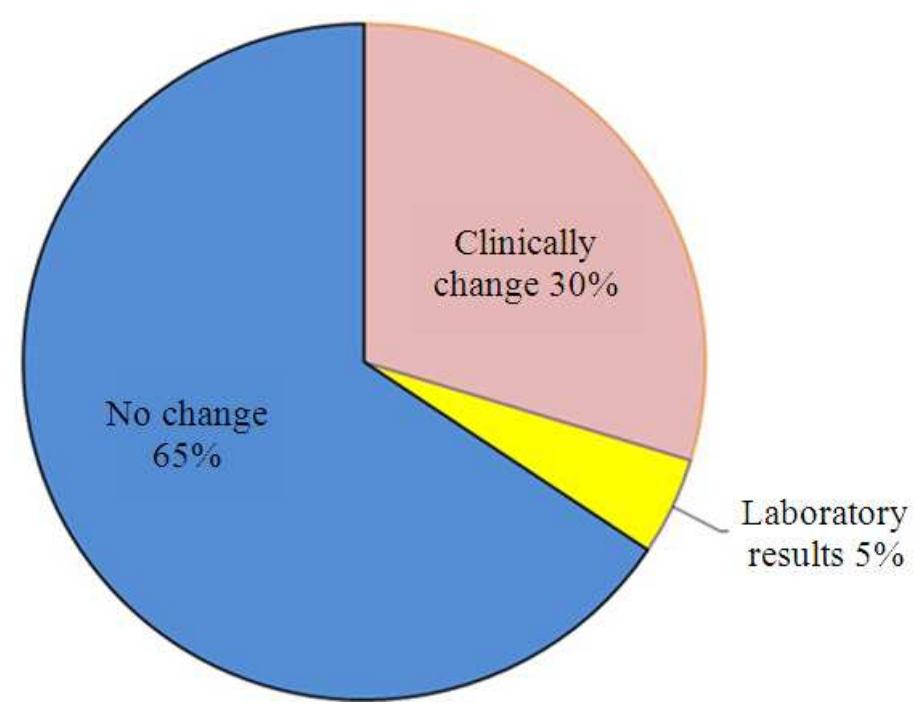

Fig. 3. The frequency of change of antibiotics during the course of treatment and base of changes

Table 1. Use of different antibiotic groups studied ICUs

\begin{tabular}{lcccrr}
\hline & Surgery & Neurology & \multicolumn{2}{c}{ Pulmonary } & \multicolumn{2}{c}{ Neurosurgery } \\
Antibitic family & ICU & ICU & disease ICU & ICU & Total \\
\hline Cephalosporin & 144 & 12 & 18 & 33 & 207 \\
Carbapenems & 12 & 3 & 15 & 6 & 36 \\
Beta-lactamase resistant penicillins & 9 & 0 & 21 & 6 & 36 \\
Aminoglycoside & 15 & 3 & 9 & 3 & 21 \\
Macrolide & 15 & 6 & 18 & 3 & 36 \\
Fluoroquinolones & 9 & 3 & 9 & 3 & 33 \\
Targosid & 3 & 0 & 90 & 60 & 384 \\
Total & 207 & 27 & & & 3 \\
\hline
\end{tabular}




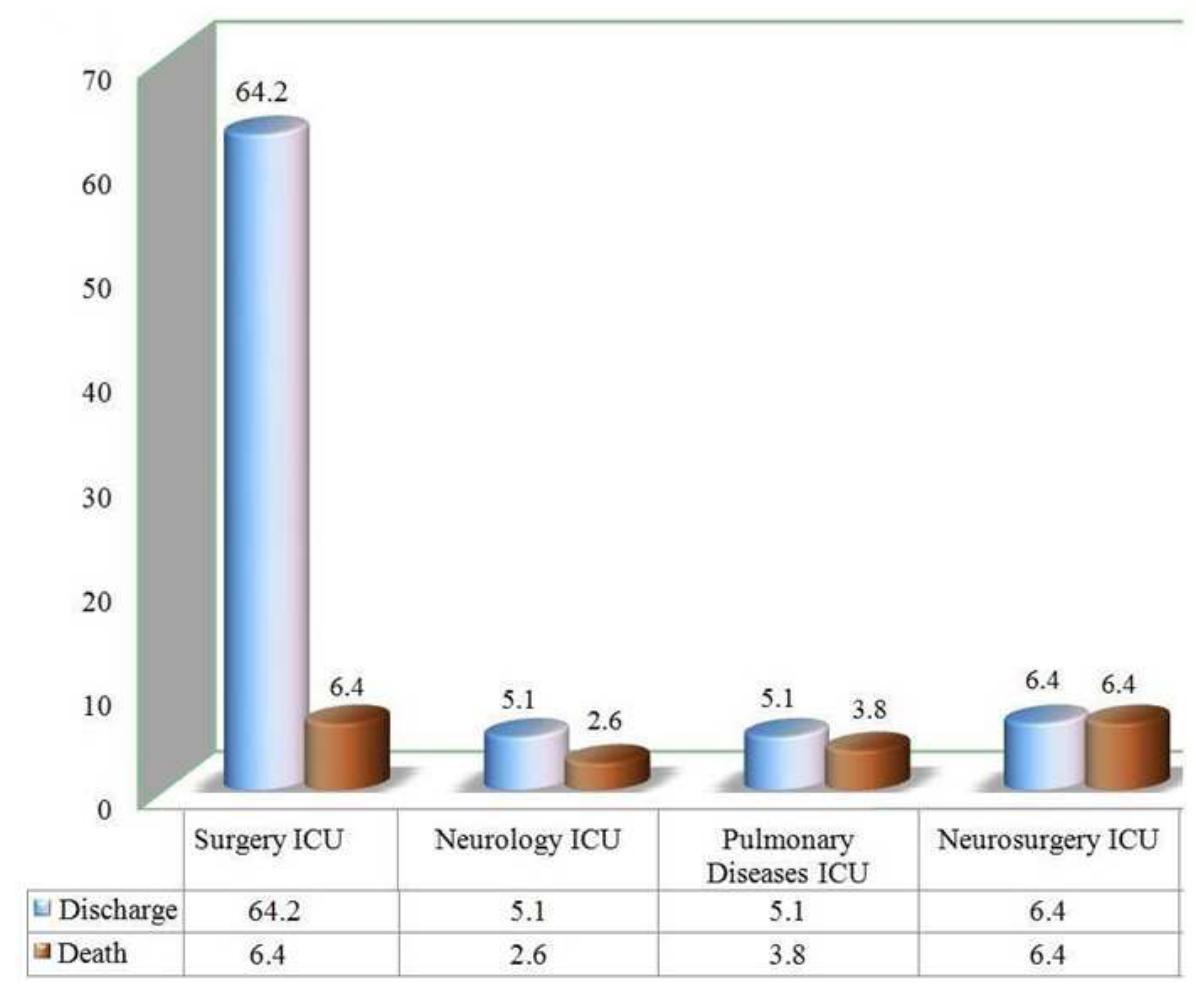

Fig. 4. Outcome and mortality of patients

Table 2. Types of used antibiotic in studied ICU wards

\begin{tabular}{|c|c|c|c|c|c|}
\hline Antibiotic family & $\begin{array}{l}\text { Surgery } \\
\text { ICU }\end{array}$ & $\begin{array}{l}\text { Neurology ICU } \\
\text { ICU }\end{array}$ & $\begin{array}{l}\text { Pulmonary } \\
\text { disease ICU }\end{array}$ & $\begin{array}{l}\text { Neurosurgery } \\
\text { ICU }\end{array}$ & Total \\
\hline Cefazolin & 66 & 6 & 0 & 3 & 75 \\
\hline Cefepim & 3 & 0 & 0 & 9 & 12 \\
\hline Cefriaxone & 75 & 6 & 6 & 18 & 105 \\
\hline Ceftazidim & 0 & 0 & 12 & 3 & 15 \\
\hline Meropenem & 6 & 3 & 0 & 3 & 12 \\
\hline Imipenem & 6 & 0 & 15 & 3 & 24 \\
\hline Tazocin & 6 & 0 & 12 & 3 & 21 \\
\hline Ampicillin sulbactam & 3 & 0 & 9 & 3 & 15 \\
\hline Amikacin & 3 & 0 & 0 & 3 & 6 \\
\hline Gentamycin & 12 & 3 & 0 & 0 & 15 \\
\hline Clinadamycin & 6 & 6 & 9 & 6 & 27 \\
\hline Azithromycin & 9 & 0 & 0 & 0 & 9 \\
\hline Ciptrofloxacin & 9 & 3 & 18 & 3 & 33 \\
\hline Targosid & 3 & 0 & 9 & 3 & 15 \\
\hline Total & 207 & 27 & 90 & 60 & 384 \\
\hline
\end{tabular}

In $35 \%$ of cases the antibiotic changed, in $30 \%$ of these cases the changes was clinical and in the remaining $5 \%$, the change was done upon the antibiogram results. In 30 cases, a consult was requested with infectious diseases specialist that there was consistency with final diagnosis in all of these patients.
The frequency of antibiotic administration and how it began is shown in Fig. 2. The frequency of these changes in antibiotics in the treatment process is shown in Fig. 3.

The rate of consumption of different antibiotic classes is shown in Table $\mathbf{1}$ and the types of antibiotics used in ICU s is shown in Table 2. 
The mean period of antibiotic administration was as below:

Surgery ICU (6.83 \pm 7.25 days), Neurology ICU $13.33 \pm 18$ days, disease ICU $25.28 \pm 27.75 \mathrm{~d}$ ays and neurosurgery ICU $12.90 \pm 17.14$ days, which it was significantly more in ICU $(\mathrm{p}<0.001)$

In $89 \%$, there was consistency in dosage of antimicrobial regimens. The mortality rate is shown in Fig. 4.

\section{DISCUSSION}

ICU is one of the most important sources of nosocomial infection and a high prevalence of infections cause a high rate of antibiotic use in this section comparing to other wards (almost 10 times more) (Thursky et al., 2006).

In our study, the most acceptances were for surgical ICU, neurosurgery ICU and Pulmonary ICU respectively.

The most common antibiotic spectrum used regardless of ICU wards, were cephalosporin's (53.9\%), followed by the carbapenem, penicillin and macrolide resistant to beta-lactamase (each 9.3\%).

ICU separated, except in the ICU where beta lactamase-resistant penicillin's was more common, cephalosporins were mostly used in other ICU wards.

The most common antibiotics used regardless of the ICU were ceftriaxone (27.3\%), cefazolin (19.5\%), ciprofloxacin (8.5\%) and clindamycin with $7 \%$.

The most common antibiotic used in general surgery ICU was Ceftriaxone (36.2\%), in neurosurgery ICU, cefazolin ceftriaxone and clindamycin with $22.2 \%$, in ICU Ciprofloxacin (20\%) and neurosurgery, ceftriaxone with $30 \%$ of usage. In a study in Italy on antimicrobial usage in ICU wards, the most common antibiotic agent used for prophylaxis was cefazolin $(22.1 \%)$, Ceftazidim $(12.4 \%)$ and ceftriaxone $(11.7 \%)$ respectively and in patients, requiring surgical intervention the most common antibiotics was Cefazolin (22.1\%) Ceftazidim (12.4\%) and Ceftriaxone (11.7\%) and in patients requiring non-surgical interventions it included ceftriaxone $(20.2 \%)$ cefazolin $(12.6 \%)$ and ceftazidime $(10.9 \%)$. Therefore, the most used antibiotic family in this study in ICU wards was Cephalosporin's. In addition in $95 \%$ of cases the antibiotic changes was upon physicians opinion without laboratory documentations.

The incidence of empirical antimicrobial therapy was $59.7 \%$ in this study, which is lower than a study conducted in 1996 on patients with pneumonia in ICU, ward (Alvarez-Lerma, 1996).
In the study above the researchers have stated that the empirical antibiotic therapy has prevented the mortality, however due to the difference in patients and ICU wards this difference can also be explained.

In another study in Italy, the empirical antibiotic therapy rate was reported as $93 \%$.

In our study, the change in antibiotic therapy was calculated as $35 \%$, which is similar to $43 \%$ of the study mentioned before (Alvarez-Lerma, 1996). In this similar study, the change in antimicrobial agents was due to antibiogram results and the unimprovement of clinical signs and symptoms, the findings of our study revealed a similar results.

In another, which was conducted to evaluate, the reasons contributing in antibiotic choice and the reasons of empirical antimicrobial therapy in ICU, the results revealed the "recommendations" as an important factor (Corona et al., 2003).

In our study, the inappropriateness of antimicrobial regimens with clinical diagnosis was $61 \%$, which is higher comparing to $29.9 \%$ of study in Washington (Ibrahim et al., 2000). The reason for this difference can be the difference in study design and the patients which in this study in $29.9 \%$ of cases there was inappropriateness of antibiotics with blood culture results while in our study the blood culture was not done for all the patients.

In our study, the rate of monotherapy was $28.1 \%$ and the rate of combination therapy was $71.9 \%$. In a study in India that is conducted to compare the monotherapy with combination therapy, the results showed that combination therapy in both empirical and therapeutic methods could effectively reduce the mortality and the ICU hospitalization (Kumar et al., 2010).

In present study the maximum duration of antibiotic therapy was related to ICU (25.3 days) followed by neurology ICU (13.3 days) and neurosurgery ICU (12.9 days). There was no similar study in this regard, but in a study, which had evaluated the effect of antibiotic therapy on final clinical results in patients with bacteremia, the results showed that the lengthening the time of antibiotic therapy makes no significant difference on clinical results (Havey et al., 2011).

The overall rate of empirical antibiotic therapy was $59.7 \%$ in our study and in $40.3 \%$ the antibiotic therapy was based on no clinical clues.

\section{CONCLUSION}

Upon the results of the present study, Cephalosporin's is the most common antibiotic class and Ceftriaxone was the most commonly used 
antibiotic in ICU sections of Tabriz Imam Reza hospital. Most of antibiotics were used just based on clinical experiences of physicians and the beginning of antimicrobial therapy was not based on microbiological results. Unfortunately, in $40.3 \%$ of cases, the antimicrobial therapy was without the clinical signs and symptoms, which are a concerning, issue because this is not the empirical administration even. The combination therapy rate was more than monotherapy. The maximum duration for antibiotic therapy was for patients in ICU, followed by neurology ICU.

\subsection{Suggestions}

Conducting another study in a longer period and with greater sample size for a more detailed evaluation. Conducting an investigation to determine the impact of infectious disease specialists consult for advice on reducing inappropriate prescribing of antibiotics.

\section{REFERENCES}

Alvarez-Lerma, F., 1996. Modification of empiric antibiotic treatment in patients with pneumonia acquired in the intensive care unit. ICU-Acquired Pneumonia Study Group. Intensive Care Med., 22: 387-394. DOI: 10.1007/BF01712153

Corona, A., G. Bertolini, A.M. Ricotta, A. Wilson and M. Singer, 2003. Variability of treatment duration for bacteraemia in the critically ill: A multinational survey. J. Antimicrob. Chemother., 52: 849-852. DOI: $10.1093 / \mathrm{jac} / \mathrm{dkg} 447$

Diaz-Martin, A., M.L. Martinez-Gonzalez, R. Ferrer, C. Ortiz-Leyba and E. Piacentini et al., 2012. Antibiotic prescription patterns in the empiric therapy of severe sepsis: Combination of antimicrobials with different mechanisms of action reduces mortality. Crit. Care, 16: R223-R223. DOI: 10.1186/cc11869

Havey, T.C., R.A. Fowler and N. Daneman, 2011. Duration of antibiotic therapy for bacteremia: A systematic review and meta-analysis. Crit. Care, 15: 267-267. DOI: $10.1186 / \mathrm{cc} 10545$

Ibrahim, E.H., G. Sherman, S. Ward, V.J. Fraser and M.H. Kollef, 2000. The influence of inadequate antimicrobial treatment of bloodstream infections on patient outcomes in the ICU setting. Chest, 118: 146-155. DOI: 10.1378/chest.118.1.146
Kolle, M.H., 2001. Optimizing antibiotic therapy in the intensive care unit setting. Crit. Care, 5: 189-195. DOI: 10.1186/cc1022

Krivoy, N., W.A. El-Ahal, Y. Bar-Lavie and S. Haddad, 2002. Antibiotic prescription and cost patterns in a general intensive care unit. Pharmacy Pract., 5: 6773.

Kumar, A., R. Zarychanski, B. Light, J. Parrillo and D. Maki et al., 2010. Early combination antibiotic therapy yields improved survival compared with monotherapy in septic shock: Apropensitymatched analysis. Crit. Care Med., 38: 1773-1785. DOI: 10.1097/CCM.0b013e3181eb3ccd

Leibovici, L., I. Shraga, M. Drucker, H. Konigsberger, Z. Samra, S.D. Pitlik, 1998. The benefit of appropriate empirical antibiotic treatment in patients with bloodstream infection. J. Int. Med., 244: 379-386. DOI: 10.1046/j.1365-2796.1998.00379.x

Rello, J., 2007. Importance of appropriate initial antibiotic therapy and de-escalatio in the treatment of nosocomial pneumonia. Eur. Respir Rev., 16: 3339. DOI: $10.1183 / 09059180.00010302$

Rice, L.B., 2003. Controlling antibiotic resistance in the ICU: Different bacteria, different strategies. Cleveland Clin. J. Med., 70: 793-800. DOI: 10.3949/ccjm.70.9.793

Roder, B.L., S.L. Nilsen, P. Magnussen, A. Enquist and N. Frimodt-Moller, 1993. Antimicrobial practice antibiotic usage in intensive care unit in a Danish university hospital. J. Antimicrob. Chemother., 32: 633-642. DOI: 10.1093/jac/32.4.633

Thursky, K.A., K.L. Buising, N. Bak, L. Macgregor and A.C. Stree et al., 2006. Reduction of broadspectrum antibiotic use with computerized decision support in an intensive care unit. Int. J. Q. Health Care, 18: 224-231. DOI: 10.1093/intqhc/mzi095

Vincent, J.L., J. Rello, J. Marshall, E. Silva and A. Anzueto et al., 2009. International study of the prevalence and outcomes of infection in intensive care units. JAMA, 302: 2323-2329. DOI: 10.1001/jama.2009.1754 